\title{
DECOMPOSITION OF CUT LOCI
}

\author{
RICHARD L. BISHOP ${ }^{1}$
}

\begin{abstract}
ABSTRACr. If $p$ is a point in a complete riemannian manifold, the points of the cut locus of $p$ are designated as singular or crdinary according to whether there is just one or more minimizing geodesics from $p$. It is proved that the ordinary cut-points are dense in the cut locus.
\end{abstract}

1 Introduction. Throughout this paper we will consider a complete riemannian manifold $M$ of dimension $d$. For $m \in M$, a cut-point of $m$ is a point $p$ such that every extension of a distance-minimizing segment from $m$ to $p$ beyond $p$ is no longer minimizing. In the tangent space $M_{m}$, a vector $x \in M_{m}$ is a tangent cut-point if the ray $t \rightarrow t x, 0 \leqslant t \leqslant 1$, is mapped by $\exp _{m}$ to a minimizing segment from $m$ to a cut-point $\exp _{m} x$. If there are two or more minimizing segments from $m$ to $p$, we define $p$ to be an ordinary cut-point. If there is just one minimizing segment, we call $p$ a singular cut-point. Ordinary and singular tangent cut-points are defined so that they correspond to the same kind of points in $M$ under $\exp _{m}$.

MAIN THEOREM. The ordinary cut-points of $m$ are dense in the cut-locus of $m$ and the ordinary tangent cut-points are dense in the tangent cut-locus.

The proof of this theorem will be completed in §4. We were led to the formulation of it in an inquiry about the smoothness properties of distance functions, and we comment on this in $\$ 2$. Our proof of the main theorem involves a similar theorem about the conjugate locus due to $\mathrm{F}$. W. Warner [3]. The results of Warner are quoted in $\$ 3$ along with some of his argument, so that we may indicate what modifications are necessary to see that they remain true for the first conjugate locus.

There is a well-known characterization of cut-points which makes it clear that both relations "is an ordinary cut-point of" and "is a singular cut-point of" are symmetric. Namely, $p$ is a cut-point of $m$ if and only if either there are two or more minimizing segments from $m$ to $p$ or there is just one minimizing segment along which $p$ is a conjugate point of $m$ [1, p 237]. In particular, a singular cut-point is always a conjugate point. This is one reason why Warner's result are pertinent, but we also need Warner's analysis to handle

Presented to the Society, November 6, 1976; received by the editors June 23, 1976 and, in revised form, December 2, 1976.

AMS (MOS) subject classifications (1970). Primary 53C20

Key words and phrases. Riemannian geometry, cut locus, conjugate locus.

${ }^{1}$ Research supported by an NSF Grant. 
the case where ordinary cut-points happen to be conjugate.

2. Smoothness of distance functions. Let $r: M \rightarrow R$ be the distance from $m$. In a neighborhood of $m, r$ is smooth except at $m$ and its gradient is a unit vector pointing away from $m$ along the minimizing segments from $m$.

Proposition. $r$ is not $C^{1}$ at the ordinary cut-points of $m$.

Proof. At an ordinary cut-point there are two contrary values for grad $r$, specifically, the limit of grad $r$ as we approach $p$ along two different minimizing geodesics.

The smoothness properties of $r$ at a singular cut-point can be more complicated. We can make calculations, as indicated later in this paragraph, on surfaces, which show that $r$ can be $C^{1}$ but not $C^{2}$. It seems likely that this is the behavior whenever the singular cut-point is the tip of a semi-cubical curve included in the first conjugate locus on a surface, but the methods of calculation used make this clear only in the case where the metric is flat in a neighborhood of the cusp. Starting with the equation $y^{3}=x^{2}$ for the cusp, the orthogonal trajectories of its tangent lines can be calculated by the usual method from elementary differential equations. The result is that the orthogonal trajectory through the origin has the form $y=c x^{4 / 3}+\ldots, c \neq 0$. We claim that the orthogonal trajectories can be made to be the level curves of a distance-function $r$ on a compact surface. We first blend together the field of tangent lines to the cusp with a field of geodesics radiating from a point $m$, by the method of Gluck and Singer [2]. The geodesic arc from $n$ to the tip is minimizing in some neighborhood of the arc, and we can map this neighborhood diffeomorphically into a compact surface. The induced metric on the image can be extended to the whole surface, and by a conformal magnification on an annulus surrounding the segment we can insure that the segment will remain minimizing.

We conjecture that for every metric on a surface $M$ and $m \in M$ there is a nearby metric such that the exponential map at $m$ of the nearby metric is a Whitney-excellent map; that is, the conjugate locus consists only of folds and semi-cubical cusps. It is difficult to see how this could fail to be true since it is known that the excellent maps form a residual set in the space of all maps, and the above example shows that there is no obstruction to an exponential map possessing the generic behavior.

In light of these remarks and the main theorem, the best that we can claim is that the closure of the set of nonsmooth points of $r$ equals the cut-locus of $m$ along with $m$ itself.

3. Warner's theorems on the conjugate locus. Warner's theorems concern the tangent conjugate locus. A regular conjugate point of $m$ is a vector $x \in M_{m}$ such that $d \exp _{m}$ is singular at $x$ and there is a neighborhood $U$ of $x$ for which the intersection of every ray (from the origin) with $U$ has just one conjugate point on it. The other conjugate points in $M_{m}$ are said to be 
singular. It is clear from the definition that the regular conjugate points form an open subset of all the conjugate points. Let $C_{r}$ denote this open subset.

THEOREM A [3]. The regular conjugate points form an imbedded smooth $(d-1)$-dimensional submanifold of $M_{m}$ which is an open dense subset of the conjugate locus.

We reproduce a part of the proof so that we can see that the same theorem is true if we replace "conjugate" by "first conjugate" throughout. Aside from the question of density this extension is obvious.

Let $x$ be a singular first conjugate point. Designate the nullity of $d \exp _{m}$ at $y \in M_{m}$ by $\mu(y)$. For a singular conjugate point $s$ it is clear that $\mu(x)>1$, because when $\mu(y)=1$ the neighboring rays to the one through $y$ can have only one conjugate point of index 1 in some neighborhood of $y$. In any neighborhood $U$ of $x$ there is a ray intersecting $U$ on which there are two or more conjugate points. The nearest to 0 , say $x^{\prime}$, is a first conjugate point. Also $\mu\left(x^{\prime}\right)<\mu(x)$, because the sum of indices on a ray is a local constant. Repeating this if necessary, we obtain a first conjugate point in $U$ which is regular.

THEOREM B [3]. At a regular conjugate point $x$ for which $\mu(x)>1$, there is a $C_{r}$-neighborhood of $x$ on which $\mu$ is constant. Moreover, the nullspace $N(x)$ of $d \exp _{m}$ at $x$ is tangent to $C_{r}$, the subbundle $N$ of $T C_{r}$ formed by these nullspaces is involutive, and the leaves of $N$ are mapped to constants under $\exp _{m}$.

THEOREM C [3]. (a) At points of $C_{r}$ for which $N$ is tangent to $C_{r}$ in some $C_{r}$-neighborhood $U$, the exponential map has the following canonical form, where the $x_{i}$ are coordinates, on $M_{m}$ and the $y_{i}$ are coordinates on $M$ :

$$
\begin{aligned}
& y_{i} \circ \exp _{m}=x_{i} \text { for } i=1, \ldots, d-\mu, \\
& y_{i} \circ \exp _{m}=x_{1} x_{i} \text { for } i=d-\mu+1, \ldots, d .
\end{aligned}
$$

(b) At points of $C_{r}$ for which $N$ is transverse to $C_{r}$, the exponential map has the canonical form of a fold:

$$
\begin{aligned}
& y_{i} \circ \exp _{m}=x_{i} \text { for } i=1, \ldots, d-1, \\
& y_{d} \circ \exp _{m}=x_{d}^{2} .
\end{aligned}
$$

(c) The points of $C_{r}$ which are neither of type (a) nor (b) are nowhere dense in $C_{r}$.

REMARKS. Warner proves these theorems in a generality which includes Finsler spaces. An immediate corollary is that $\exp _{m}$ is not injective in a neighborhood of a conjugate point.

4. Proof of the main theorem. It suffices to prove the tangent cut-point version since a dense subset will be mapped to a dense subset by the continuous map $\exp _{m}$.

The distance to the tangent cut-point is a continuous function of the ray on which it occurs [1, p. 239], and the conjugate locus is closed. It follows that a 
nonconjugate ordinary tangent cut-point has a neighborhood in the tangent cut-locus consisting of the same kind of points. Thus, for the remainder of the proof we may concern ourselves with tangent cut-points which are also first conjugate points.

Suppose that $x \in M_{m}$ is a cut-point which is a conjugate point of type (a) in Theorem $\mathrm{C}$. Then the leaf of $N$ in $C_{r}$ through $x$ is mapped to the same point in $M$. The nullspaces of $\exp _{m}$ are always orthogonal to the rays from 0 so that the rays to the leaf through $x$ all map to minimizing segments. Thus, every cut-point of type (a) is an ordinary cut-point.

Suppose that $x \in M_{m}$ is a cut-point which is a conjugate point of type (b) in Theorem $\mathrm{C}$. Then by the coordinate form for $\exp _{m}$ in a neighborhood $U$ of $x$, the image of $U$ is a half-ball $y_{d} \geqslant 0$, with conjugate points on the boundary. Let $p=\exp _{m} x$, and let $\left(p_{i}\right)$ be a sequence converging to $p$ and lying in the half-plane $y_{d}<0$. We can take the $p_{i}$ to be so close to $p$ that every minimizing segment from $m$ to a $p_{i}$ cannot have its initial tangent falling in a fixed open cone about the ray to $x$. There will be a convergent subsequence of such minimizing segments, whose limit must be a different minimizing segment to $p$ than the one generated by $x$. Thus, every cut-point of type (b) is also an ordinary cut-point.

Finally, suppose that $x \in M_{m}$ is a cut-point which is a conjugate point, but not of types (a) or (b). By Theorems $\mathrm{A}$ and $\mathrm{C}$, the first conjugate points of types (a) and (b) form a dense subset of the first conjugate locus. Hence, $x$ is a limit of a sequence $\left(x_{i}\right)$, each of which is a first conjugate point of type (a) or (b). Let $y_{i}$ be the cut-point on the ray $t \rightarrow t x_{i}, 0 \leqslant t \leqslant 1$. If $y_{i}=x_{i}$, then we have seen that $y_{i}$ is an ordinary cut-point. Also if $y_{i} \neq x_{i}$, then $y_{i}$ is not a conjugate point and hence is an ordinary cut-point. The sequence of rays of $\left(x_{i}\right)$ converges to the ray of $x$, so that $\left(\left\|y_{i}\right\|\right)$ converges to $\|x\|$ and $\left(y_{i}\right)$ converges to $x$. This proves that the ordinary cut-points are dense in the cut-locus.

REMARK. We have not been able to prove that the ordinary cut-locus is open in the cut-locus, either in $M_{m}$ or in $M$. It is clear that the nonconjugate tangent cut-points and the conjugate cut-points of type (a) or (b) are interior points of the ordinary tangent cut-locus.

\section{REFERENCES}

1. R. L. Bishop and R. J. Crittenden, Geometry of manifolds, Academic Press, New York, 1964. MR 29 \#6401

2. H. Gluck and D. Singer, Deformations of geodesic fields, Bull Amer. Math. Soc. 82 (1976), 571-574.

3. F. W. Warner, The conjugate locus of a Riemannian manifold, American J. Math. 87 (1965), 575-604. MR $34 \# 8344$

Department of Mathematics, University of Illinois at Urbana-Champaign, Urbana, ILLNOIS 61801 